\title{
Individualized Intervention to Support Mental Health Recovery Through Implementation of Digital Tools into Clinical Care: Feasibility Study
}

\author{
Elizabeth Carpenter-Song ${ }^{1} \cdot$ Stephanie C. Acquilano ${ }^{2} \cdot$ Valerie Noel $^{3,4} \cdot$ Monirah Al-Abdulmunem $^{5} \cdot$ John Torous $^{6}$. \\ Robert E. Drake ${ }^{5}$
}

Received: 15 September 2020 / Accepted: 8 February 2021 / Published online: 21 February 2021

(c) The Author(s), under exclusive licence to Springer Science+Business Media, LLC part of Springer Nature 2021

\begin{abstract}
Myriad digital tools exist to support mental health but there are multiple barriers to using these tools in routine care. This study aimed to assess the feasibility of an intervention incorporating a support role to help the clinical team identify and use technology to promote recovery. The technology specialist intervention is 3 months in duration and comprises four stages: goal setting, researching and evaluating tools, demonstrating and selecting tools, and ongoing support. We implemented the intervention in a community mental health center and a dual diagnosis treatment program, working with eight clients and their case managers. Clients and case managers willingly engaged with the technology specialist and found the intervention beneficial. Integration and collaboration with the care team facilitated implementation of the technology specialist in these real-world settings. Clients reported that the intervention made it easy to try a digital tool. Six of the eight participants stated that they made substantial progress toward their goals. The technology specialist is a promising new role for mental health care delivery to augment traditional services and enhance individualized recovery.
\end{abstract}

Keywords Qualitative research · Technology $\cdot$ mHealth $\cdot$ Digital health $\cdot$ Apps $\cdot$ Mental health $\cdot$ Smartphone $\cdot$ Patientcentered intervention

\section{Introduction}

Even before the COVID-19 pandemic, recognition of the potential of using technology, especially mobile technology, to support mental health (mHealth) was quickly increasing (Ben-Zeev et al. 2020; Buck et al. 2020; Lecomte et al. 2020;

Elizabeth Carpenter-Song

Elizabeth.A.Carpenter-Song@Dartmouth.edu

1 Department of Anthropology, Dartmouth College, Hanover, $\mathrm{NH}, \mathrm{USA}$

2 The Dartmouth Institute for Health Policy and Clinical Practice, Geisel School of Medicine at Dartmouth, Lebanon, $\mathrm{NH}$, USA

3 ACCESS Open Minds (Pan-Canadian Youth Mental Health Services Research Network), Montréal, QC, Canada

4 Douglas Hospital Research Centre, Montréal, QC, Canada

5 Westat, Lebanon, NH, USA

6 Division of Digital Psychiatry, Beth Israel Deaconess Medical Center, Harvard Medical School, Boston, MA, USA
Santarossa et al. 2018). Several factors contributed to this recognition, including the increase in smartphone ownership among individuals with mental illness (Firth et al. 2016; Pew Research Center 2018; Young et al. 2020), proliferation of available health and mental health apps (IQVIA 2017), and strong interest in and acceptability of mobile technology use by individuals living with mental illness (Carpenter-Song 2020; Carpenter-Song et al. 2018; Firth et al. 2016; Jonathan et al. 2019; Noel et al. 2019a, b; Roberts et al. 2018; Santarossa et al. 2018). Yet recognition of the potential benefits of technology alone has not transformed mental health care.

Researchers have developed a wide range of mHealth digital tools to support mental health, including those to improve patient-provider communication and shared decision making, help individuals cope with mental health symptoms, provide ongoing (self-report and passive sensing) monitoring of mental health symptoms, and provide interventions to address serious mental illnesses (Ben-Zeev et al. 2014, 2017, 2020; Biagianti et al. 2017; Deegan 2010; Gammon et al. 2017; Garety et al. 2017; Jonathan et al. 2019; Roberts et al. 2018; Schlosser et al. 2016; Torous et al. 
2015). Accumulating evidence indicates that some technology-delivered interventions may be as effective as in-person interactions (Ben-Zeev et al. 2018; Gire et al. 2017; Hubley et al. 2016; Lecomte et al. 2020; Santarossa et al. 2018; Wright et al. 2019). Telepsychiatry and mHealth show promise in addressing disparities in access to care such as those experienced by people living in rural or other low-resource settings (Biagianti et al. 2017; Gire et al. 2017; Mahmoud et al. 2020; Torous et al. 2017a, b) and may improve engagement and retention for some of the highest risk patients (Ben-Zeev et al. 2016; Buck et al. 2020).

Despite evidence regarding the potential benefits of mHealth, adoption and implementation of digital tools in routine mental health care settings is lacking (Chiauzzi and Newell 2019; Noel et al. 2019a, b; Wisniewski and Torous 2020). First, the sheer number of existing health-related apps poses serious challenges in terms of finding tools that are consistent with research evidence, easy to use, and secure (Lecomte et al. 2020). Clinicians are often unsure of their capability to select and use mHealth tools (Waalen et al. 2019) and many clients find these tools challenging to use (Wisniewski and Torous 2020). Typical clinical workflows make the integration of digital tools difficult, implementation efforts are often seen as burdensome to clinicians, and various concerns regarding cost are common (Gagnon et al. 2016). Effective support to improve clinician and client adoption and meaningful use of digital tools is critical and lacking (Waalen et al. 2019).

In addition to implementation challenges, the accumulating evidence of the potential benefits of digital tools to support mental health is counterbalanced by concerns regarding potential harms. Researchers have identified mental health apps that contain information inconsistent with current practice guidelines (Nicholas et al. 2015) and others have raised concerns regarding the validity of evidence used to support claims of efficacy in mental health apps (Cosgrove et al. 2020). Particularly concerning, some apps have been found to contain potentially harmful information (Larsen et al. 2016). In addition, scholars have identified ethical questions raised by the turn toward digital technologies, including privacy of data shared with third-parties as well as potential commodification of vulnerable populations (Cosgrove et al. 2020; Zuboff 2019).

To address the myriad challenges involved in digital mental health tools, experts have recommended technology supports and identified the need for a unique support role to aid in this area (Ben-Zeev et al. 2015; Wisniewski and Torous 2020). Responding to this need, our team developed the Technology Specialist intervention (Carpenter-Song 2020; Noel et al. 2019a, b). The Technology Specialist works collaboratively with clients and clinicians to integrate available technology-based tools into routine mental health care to support clients' individualized recovery goals. Our intervention is designed to create opportunities for those with mental illnesses to have discussions with the Technology Specialist and their clinical team about digital technology, including issues regarding privacy and security. Our previous research found strong interest in using technology to support mental health, yet technology was rarely discussed in the context of routine clinical encounters (Carpenter-Song et al. 2018). Absent such discussions, clients were on their own to navigate the complex and potential harmful landscape of digital technology. The technology specialist, as an integrated role within mental health services, may support informed choices about the use of technology and encourage ongoing attention to, and discussion of, the use of technology in routine care.

This article reports findings from a feasibility study of the technology specialist intervention at two mental health care settings in New England. The study addressed the following key research questions:

(1) Would mental health clients and clinical team members engage with a Technology Specialist?

(2) Do clients and clinical team members perceive a benefit from working with a Technology Specialist?

(3) What are the barriers to, and facilitators of, integrating the Technology Specialist into mental health treatment settings?

\section{Methods}

\section{Participants}

This study included individuals with serious mental illness receiving outpatient services at one of the two participating clinics. Eligibility criteria were: aged 18 years or older, owned a smartphone with a data plan, and both the client and their clinician were willing to take part in the study. Clients were excluded if they were experiencing an acute symptom exacerbation or medical illness, did not speak English, or were unable to provide informed consent. The final sample included eight participants $(7 \mathrm{men}$, 1 woman) with serious mental illness. They ranged in age from 25 to 52 years $(\mathrm{M}=33.2, \mathrm{SD}=9.7)$; all participants were White/non-Hispanic and had at least a high school education or equivalent. Most participants had never been married $(\mathrm{n}=7,88 \%)$ and half $(\mathrm{n}=4,50 \%)$ were unemployed; $2(25 \%)$ were employed part-time and $2(25 \%)$ were employed full-time. Conducting exploratory feasibility research with a small sample size follows recommendations for developing evidence-based interventions (Mueser and Drake 2005). 


\section{Procedures}

All authors certify their responsibility for the manuscript and report no financial relationships with commercial interests. The Dartmouth College Committee for the Protection of Human Subjects and the New Hampshire Department of Health and Human Services Committee for the Protection of Human Subjects approved this study, which followed the principles outlined in the Helsinki declaration. All participants provided informed consent. We recruited participants from two mental health centers: one dual diagnosis treatment program that serves men with co-occurring serious mental illness and substance use disorder and one community mental health center that serves adults with serious mental illness.

Case managers identified clients who expressed interest in the project. The Technology Specialist met with the case manager and the client together to review the purpose of the study, answer questions, and obtain consent to participate.

\section{Technology Specialist Intervention}

The technology specialist intervention is designed to promote progress toward individual recovery goals by identifying and evaluating technology-based supports that reflect best practices and minimize privacy and security risks. The digital tools considered within scope for this feasibility study were self-management tools that aim to support the development of behaviors/habits for general wellness (e.g., nutrition, smoking cessation), support new skills to manage mental health symptoms (e.g., meditation), or inform awareness of mental health symptoms (e.g., mood trackers). The technology specialist must have a basic foundational understanding of mental illness, treatment approaches, and signs of crisis, as well as a keen interest in technology, natural curiosity, and strong interpersonal skills. The technology specialists in this project had advanced degrees in psychology, a great deal of research experience, and minimal experience providing clinical services. During the intervention, the technology specialist leverages the strengths of existing digital tools, following six principles:

- "Accessibility" All clients who express interest and have an internet-enabled device are eligible for this intervention. Clients do not need to be adept with technology; we expect that clients will have a range of knowledge and experience with technology. The technology specialist works with people at their ability and comfort level. For example, one client was extremely inexperienced with technology and struggled to even answer phone calls consistently, while another wanted to use two apps, one of which was a sophisticated budget management app. Our approach with each reflected their level of interest and experience.

- "Integration" The technology specialist works to integrate technology tools as supports for recovery goals in collaboration with the client's care team. This approach aims to enhance and extend, rather than replace, the work of mental health professionals. For example, clinicians and clients are strongly encouraged to include discussions of the recovery goal and how well the technology tool is supporting that goal in their regularly-scheduled clinical visits.

- "Focus on Recovery" Supporting people in their recovery is the goal of the intervention. Digital tools are viewed as resources to aid clients in making progress on recovery goals. Use of digital tools is not an end in itself, but rather in the service of supporting recovery. For example, in discussions with clients, the technology specialist and the clinician ask about progress toward the recovery goal itself, not how often the client is using the tool. Any specific questions about the tool revolve around whether and how it is helping with the recovery goal. If a specific tool is not perceived to be supporting the recovery goal, the Technology Specialist works with the client and clinician to identify other digital tools that may better meet the client's needs and preferences.

- "Focus on the Individual" The technology specialist identifies digital tools and tailors the intervention to meet the client's individualized needs and recovery goals. For example, the technology specialist gathers detailed information about the recovery goal. While several people might express similar goals (e.g., wanting to meditate regularly), most will have unique caveats such as also wanting to journal, preferring very brief meditations as opposed to longer ones, wanting to meditate as part of a daily routine versus as-needed for stress. The intervention uses all of this information to identify potential digital tools for each individual.

- "Shared Decision-Making" The client, clinician, and technology specialist collaboratively discuss recovery goals and potential digital tools. Client preferences are elicited and guide decisions throughout the process of selecting digital tools. For example, the conversation begins with the Technology Specialist asking the client to describe their recovery goals and this serves as the starting point for a collaborative discussion about which goal might fit best for the client and for inclusion in the clinical relationship.

- "Creativity" The intervention takes a broad view of what constitutes "technology" (e.g., mobile apps, websites, wearables) and considers a wide range of tools that may be helpful, not just those originally intended as healthrelated. For example, someone who has difficulty managing high-emotion situations with relaxing breathing tech- 
niques may enjoy a brain teaser or puzzle app to distract attention away from the source of the distress.

The intervention comprises four stages: Goal setting, researching and evaluating tools, demonstrating and selecting tools, and ongoing support.

\section{Goal Setting}

The initial step in the intervention is a meeting between the technology specialist, client, and clinician to discuss the client's recovery goals and identify a goal for which the client would like additional support. This meeting is a collaborative, shared decision-making conversation guided by the client's values, needs, and preferences. The clinician provides input regarding integrating the goal in the client's treatment and the technology specialist discusses relevant resources. This meeting may occur in-person or virtually, preferably via videoconferencing to enhance relationship development, typically takes 30-60 min, and culminates with the group agreeing on a recovery goal to pursue. The driving influence here is the client's preference; the clinician and technology specialist must remember that there are multiple goals from which to choose and the needs, values, and beliefs of the client take precedence over other factors. Disagreements about which goal is most appropriate may occur and typically involve some sort of miscommunication. For example, the clinician may not fully understand the client's preferences or the client may not fully understand the circumstances around choosing a particular goal (Zeuner et al. 2015). Either way, resolving the disagreement and choosing a goal will involve further discussion to better understand each person's perspective in pursuit of a choice that is ultimately best for the client (Montori et al. 2006; Zeuner et al. 2015). This careful discussion and consideration may not only lead to the best choice for the client but may also serve to strengthen the client-clinician relationship through enhanced understanding of each other (Montori et al. 2006). During the meeting the technology specialist gathers basic information about what technology devices the client owns, any limits on internet access or data usage, and experience with and preferences in technology.

\section{Researching and Evaluating Tools}

Based on information obtained during the goal setting meeting, the technology specialist begins to explore a wide variety of technology resources that may be appropriate. The technology specialist must stay creative and cast a wide net in order to identify up to 10 potential resources for further evaluation. Web searches using a variety of phrases related to the goal will identify numerous sets of potential sources from which to begin selecting promising tools. Tools that are recommended by multiple sources are often a good place to start. For example, one participant had never meditated but wanted to start, partly in an effort to manage stress. We conducted searches using phrases like "best apps for meditation," "best meditation apps for beginners," and "learning to meditate." These searches identified many lists of resources, several of which recommended Aura, Headspace, Calm, Buddhify, and Stop Breathe Think.

Full evaluation of possible tools follows the evaluation framework originally created by Torous and colleagues (Torous et al. 2018), as adopted by the American psychiatric association (https://www.psychiatry.org/psychiatrists/ practice/mental-health-apps/app-evaluation-model). In brief, this framework guides evaluation of resources based primarily on privacy and security, quality of evidence or clinical foundation, usability, and capacity to integrate with other resources (when relevant). This step leads to the identification of 2-4 technology resources to present to the client and clinician. A careful review of the terms of service and privacy policy will provide the necessary information regarding privacy and security; we do not recommend using apps or websites that have no such documents. The technology specialist evaluates usability by spending time experimenting with the tool and exploring all of its features to make a more informed determination about usability, noting characteristics such as ease of navigation and clarity of key functions and features within the tool. There are a few ways to evaluate the evidence or clinical foundation of tools. Some tools' benefits have been documented in clinical studies and a brief search of the tool name on Google Scholar, PubMed, or Ovid should identify this evidence. However many, if not most, tools have not been formally evaluated. For these tools, the technology specialist must look for independent reviews of the tools (e.g., reviews by experts not affiliated with the developer, e.g., Lifewire, Buzzfeed, or DevelopGoodHabits), recommendations by relevant national organizations (e.g., American Psychiatric Association, National Institutes of Health, Substance Abuse and Mental Health Services Administration), and summaries from academic and medical research centers (Center for Technology and Behavioral Health's Eye on Innovation, Beth Israel Deaconness Medical Center Division of Digital Psychiatry's MIND). Additional strategies include considering whether the tool's features appear to be based accurately in evidence-based principles, looking at the entity/team that developed the tool, and examining user reviews. The latter, however, should not be the sole source of evidence of quality, only a component along with other, more reliable, sources of evidence. In addition, the Technology Specialist is encouraged to lean on the clinical expertise of the team in which they are embedded for support in evaluating the quality and evidence for the tool. 


\section{Demonstrating and Selecting Tools}

The next step involves another meeting of the technology specialist, client, and clinician. The technology specialist briefly describes the process of exploring possible options, experimenting with them, and ultimately evaluating a handful to select a few to present to the client and clinician. The technology specialist provides an overview of each of the tools, describing their features, privacy issues, and functionality, and demonstrating each one. The client and clinician then experiment with the tools to see which are most appealing so that they may agree on one to try. The technology specialist facilitates this decision by leading a discussion of the client's and clinician's preferences and comfort level, exploring how well various features of the tools align with those preferences. Once the client and clinician decide on a technology tool, the technology specialist guides them through the process of downloading, installing, and setting up the tool so that when the meeting is over, both the client and the clinician are ready to use the tool without additional setup. While this meeting may occur via videoconference, it is best conducted in-person and typically takes 30-60 min.

\section{Ongoing Support}

To promote adoption of the technology tool and integration in clinical care, the technology specialist maintains contact with the client and clinician via phone calls, texts, or emails according to their stated preferences. For the first month following the selection of the tool, the technology specialist contacts the client bi-weekly. Then, scheduled contact is reduced to monthly intervals. During these contacts, the technology specialist inquires whether clients and clinicians are using the technology tool, finding it helpful in supporting the goal, experiencing any technical difficulties, and discussing it during clinical care. If the tool does not seem to be working well or supporting the recovery goal, the technology specialist offers to meet with them again to problemsolve and possibly select a different tool.

\section{Measures}

\section{Baseline}

We collected data on sex, age, race, ethnicity, education, employment, and marital status for all participants. The technology specialist maintained detailed notes regarding the identified goals, search strategies, and selected technology tools, as well as completed worksheets used during research and evaluation of potential tools.

\section{Post-Intervention Assessment}

At the end of 3 months, participants and case managers participated in a semi-structured qualitative interview (either in-person or via videoconferencing). This interview inquired about overall experience with the intervention, what worked best and what could be improved, what the process was like, how helpful the intervention was, what kind of impact (if any) it had, and whether they would continue to work with a Technology Specialist in the future or recommend it to a friend. Members of the research team who were not involved in the delivery of the intervention conducted these interviews, which were approximately $30 \mathrm{~min}$ in duration and were audio-recorded for transcription and analysis. Participants received \$20 in gift cards as compensation for completing the post-intervention assessment.

\section{Data Analysis}

We used Dedoose, a qualitative analytic software program to manage and code interview transcripts. Qualitative codes were developed on the basis of a priori categories from the interview guides as well as inductively derived categories based on immersion in the dataset (Charmaz 2014). Codes were developed iteratively and collaboratively by members of the team (ECS, SA, VN, MA) to reach consensus on the final code list applied across the dataset. Aggregated code reports were produced in Dedoose for codes relevant to feasibility and acceptability. Team members reviewed the code reports to develop the key thematic findings reported here (Charmaz 2014).

\section{Results}

\section{Mental Health Clients and Clinicians Willingly Engaged with the Technology Specialist}

Both clients and clinicians in the two mental health treatment settings were interested in working with the technology specialist. Some clients were drawn to the possibility of technology offering new approaches to supporting mental health:

I had tried more traditional methods outside of technology-based resources and when I did that I had mixed results, so I was hoping to work with her and get something that was a little off the beaten path, and she was very effective at finding a couple of things that fit for that. 
Similarly, other clients hoped to find new strategies to support specific needs or goals:

I just was hoping to find outlets to help with highstress situations that I normally wouldn't be able to find on my own. So, working with someone who had gone through a much bigger collection of applications or practices that I wouldn't have knowledge about, or they would have better knowledge about to help as a guide to better dealing with stress.

Some clients specifically noted the value of having assistance to identify technology-based tools among the wide array of available options:

I think it would be an interesting kind of thing to have on hand. Like, 'Hey I' $m$ interested in having an app that makes it easy for me to do this.' Having that resource would be really cool because it can be really overwhelming to like, 'Hey, I want something for this but, there's six thousand apps for this.

Several clinical team members spoke of technology as an under-utilized resource for recovery despite its ubiquity:

There's so much potential to utilize something. A cell phone is in the hands of every participant pretty much all the time. Why isn't [it] being utilized for their recovery?

Similarly, clinical team members expressed the need for greater support in helping clients to use technology in ways that can be beneficial to mental health:

I think every mental health facility should be addressing something about how to best utilize apps that are out there, and also what are the apps that can help people towards their goals because, ultimately, we spend a lot of [time] - I mean, the average person spends over four hours on their phone... How can we best utilize that as a tool? Not something that's going to pull us away from achieving our goals.

Other clinical team members saw value in receiving support to find tools that could help service-users outside of direct clinical care:

I think that for a lot of the [clients] it could be an extremely helpful tool to find whatever app that would be helpful for them, or multiple apps that would be able to bridge that gap between time that they're not engaged in direct recovery-related activities.

\section{Mental Health Clients and Clinicians Viewed the Technology Specialist as Beneficial}

Both clients and clinicians identified benefits to having participated with the Technology Specialist. Many clients noted that working with the technology specialist had motivated them to try something new to support their mental health:

Overall, it's shown me that it doesn't hurt to try new things.

I think it just comes down, in my instance, to trial and error and to just keep working at it and keep trying to find new ways. Because even if I don't end up using something that I found directly through this program, I think it would definitely be useful for me in order to consider something similar that I might just pick up on my own time.

Similarly, other clients specifically noted the role of the Technology Specialist in guiding the use of technology:

I'm not the most technology savvy person and I didn't have any issues because I had already been walked through it...I think I wouldn't have actually started on anything if she hadn't made it easy to do so. I don't even like apps honestly so, the fact that the process was just simple. Because, it was explained to me, the choices were laid out, I could choose between the three.

This was especially important for those clients who had limited experience using technology. One such participant expressed the profound impact of working with the technology specialist to learn basic phone skills:

It's like a whole new world with my cell phone. I couldn't even answer my phone [before]. But [now] I always get it. I don't miss phone calls.

Several clinicians found that the technology tool offered a point of departure for discussions with clients:

I thought it was exciting because it was a new way of talking to people, especially younger people who are phone and app savvy.

Similarly, some clinicians noted that the technology tool offered a way to focus clinical work with clients:

It definitely added something on my to-do list with [client] in regards to making sure that this is something he's continuing to do on a regular basis for his own recovery. He's someone that struggles with consistency and this was a good tool for me to use as a guide. It was a really positive thing for his own recovery. 
Some clinicians also noted that having a technology specialist on site heightened their awareness of how technology can augment clinical efforts to support recovery:

It kind of opened my eyes ... there is so much stuff out there that can be helpful besides your clinical team, which people might not perceive as being available $24 / 7$ even though we are. If you're picking up the phone to do something, like pick up to call a [drug] dealer or something like that, you've got this app there that's just staring you in the face, and it's just maybe an extra tool to be able to put between you and healthy decisions.

Clients across both treatment settings focused on a range of recovery goals in the context of working with the technology specialist (see Table 1). Six of the eight clients reported making substantial progress toward their recovery goals and case managers agreed that clients demonstrated differences in behavior to support those assertions. In the quote below, a client describes the impact of having new strategies to reduce stress:

So when I worked with [the technology specialist] we identified some programs or applications where I can use it. And it's just a quick, easy way to get my mind at ease. Put it at ease, focus on something else, and just relax a little bit. Some of it was successful. I definitely learned a little bit about what I need to do moving forward for that to be very useful, for the most part.

Clinical team members also noted observable changes among service-users. For example, one client's goal was to eat healthier and he used an app to help cook more nutritious meals. His case manager commented:

I was very surprised by the amount of options that were out there and so was [client]. He truly enjoyed the app that he finally picked, and he did use it for some time to help with his meal planning. He still does, to this day, use that app just to see what kind of new meals that he could do for the week. It definitely had a good turnout with him.

\section{Integration and Collaboration Facilitated Implementation of the Technology Specialist into Mental Health Treatment Settings}

Various factors facilitated the implementation of the technology specialist in both mental health settings. The technology specialist made efforts to become a familiar and trusted presence in the clinic among staff and clients. Prior to launching the intervention, the technology specialist and members of the research team met with leaders and clinical staff to collaboratively plan for implementation. Throughout the process of working with clients, the technology specialist communicated regularly with staff members and attended staff meetings to provide updates. Clinical team members were key partners in identifying potential participants and also worked collaboratively with clients to identify recovery goals. Clinicians appreciated that the technology spcecialist actively engaged with clients to understand their individual needs and goals:

I really enjoyed the assessment process and how ... I'm just thinking back to some of those initial interviews of how you two really took the time to get to know where [people] were at, listening to their stories, listening to what their troubles were and what they wanted to achieve, I think that was fantastic.

\section{Challenges and Opportunities for Implementing the Technology Specialist}

Despite efforts to become a fully integrated member of the care team, there were some challenges. In one setting, the initial enthusiasm for using technology had waned over time:

I was really encouraged about the idea of us having the technology [specialist] and really saw a lot of potential. In practice, in my experience working with the participants, I don't know how well it worked out in the long run... in recent times I just noticed some of the guys that have fallen off the whole utilizing their technology for their recovery. I'm torn, to be honest.

This sentiment was echoed by a client, pointing to the challenge of sustaining interest:

It was nice initially, but then eventually it was kind of like, life gets in the way and then you eventually kind of fall off.

A staff member in one setting expressed concern regarding the technology specialist adding burden to their team's workload:

I definitely saw reluctance in a lot of the rest of my team. I don't have time to sit with someone and discuss how they're utilizing the app to set goals or what have you. I've got too many other things going on. A part of it was us not really knowing how to do that, as well as feeling overwhelmed with the amount of things we already have going on.

Suggestions by both clients and clinicians for improving the implementation of the technology specialist emphasized the need for more frequent communication in order to sustain attention to the use of technology in supporting recovery. 


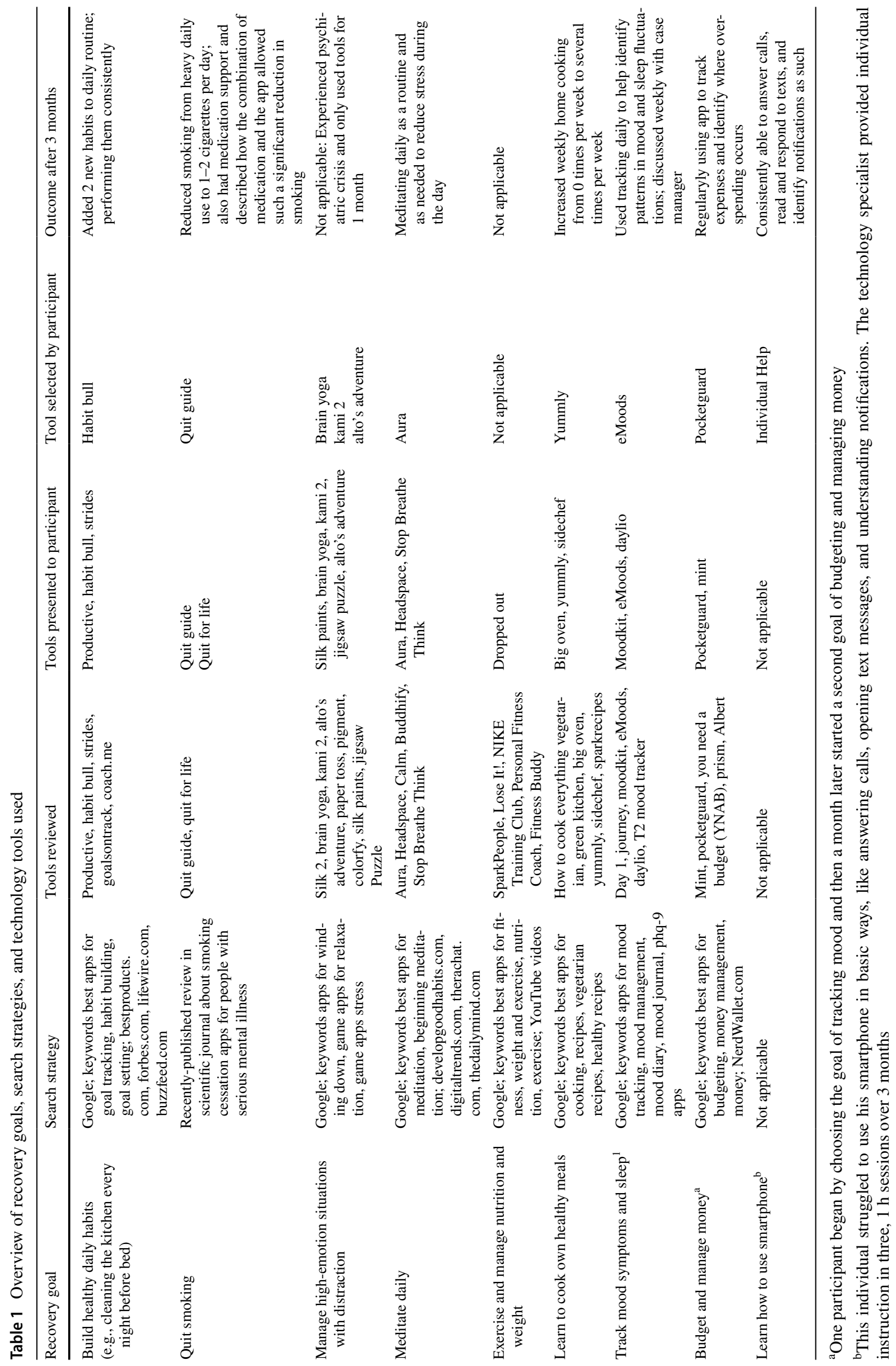




\section{Discussion}

Several findings emerged from this feasibility study. Clients expressed interest in a wide range of recovery goals, many of which did not directly involve symptoms of mental illness. They demonstrated varying levels of experience and comfort with digital tools and mobile devices; for example, one client needed basic help in simply using his phone effectively, while others were quite facile. Clients and clinicians both recognized the potential of technology tools to support recovery, within direct care and outside of treatment, but both groups lacked expertise in idenitfying and evaluating these tools. They appreciated the Technology Specialist's expert help, simplifying the process of trying something new. Many clients found that the intervention motivated them to work on a specific goal, which they wouldn't have tried without the help of the Technology Specialist. Several clinicians described that the intervention improved communication and focus in treatment. Most reported that clients made observable behavioral changes during the intervention. Clients and clinicians alike reported that the individualized approach, careful evaluatuion of tools, and collaborative nature of the intervention were key aspects of its success.

Despite these positive experiences, both groups also expressed some reservations. Clients' interest waned over the 3-month intervention. Clinicians wanted more consistent communication about clients' progress in the intervention. Some clinicians expressed ambivalence regarding whether the intervention increased or diminished work burden.

Our findings indicate that clients and clinicians are interested in accessing digital tools that may enhance clinical care and self-management toward recovery goals, but they find the task of accomplishing this daunting. They appreciated the technology specialist intervention because of the individualized approach and flexibility in range of activities. The intervention was most helpful when clinicians were able to integrate discussion of the recovery goal and technology tool into the client's clinical care visits. However, some client-clinician dyads did not manage to incorporate the goal and tool into clinical care and likely needed more structure or training to use the intervention more collaboratively. While clients still reported that the intervention was helpful in these instances, collaboration would increase utility. Sustaining interest in digital tools may require better communication between the Technology Specialist and the care team, regularly re-evaluating progress on recovery goals, potentially changing goals and tools.

Other researchers have identified the importance of clients and clinicians using technology tools collaboratively (Chiauzzi and Newell 2019; Dugdale et al. 2019; Fortuna et al. 2019; Mohr et al. 2011; Torous et al. 2017a, b) and the need to support technology use in community-based care (Hoffman et al. 2020; Jonathan et al. 2017; Wisniewski and Torous 2020). The Supportive Accountability theory proposes that involvement of a support person in promoting technology use in clinical care not only enhances the effectiveness of the technology tools but may also help sustain engagement (Mohr et al. 2011). Digital tools assist individuals with serious mental illness through a variety of mechanisms (Brunette et al. 2011; Crookston et al. 2017; Jonathan et al. 2019), several of which are included in the most common behavior change theories. For example, enhanced self-efficacy is a component of the health belief model, theory of planned behavior, transtheoretical model, and social cognitive theory (Naslund et al. 2017). We hypothesize that one of the key functions of the technology specialist intervention may be to increase clients' feelings of self-efficacy and that the Technology Specialist serves as a critical facilitator of integrating technology tools into clinical care.

We are in a unique and critical time with respect to using technology to deliver mental health services and support individuals with lived experience. The COVID-19 crisis has diminished resistance to using technology and heightened use of all forms of technology to limit in-person encounters and enhance communication. This quick adoption of technology has transformed the delivery of mental health care, which is likely to endure beyond the pandemic (Bartels et al. 2020; Ben-Zeev 2020; Goldman et al. 2020; Inkster et al. 2020; Torous et al. 2020). Leaders in behavioral health are actively advocating for expanding access to digital healthcare and supportive technologies, especially for the most vulnerable populations such as those with serious mental illness (Ben-Zeev 2020; Inkster et al. 2020; Torous et al. 2020). At the same time, movement toward increased integration of technology in mental health care needs to be approached in ways that mindfully address concerns and promote meaningful recovery (Carpenter-Song 2020). The technology specialist may be a useful strategy for promoting greater attention to technology use in routine mental health encounters and for addressing privacy and ethical concerns in real-time with clients. Supporting mental health clients and clinicians to understand more deeply about both the potential benefits and drawbacks of digital tools is a key goal of the Technology Specialist intervention.

In the translation from research to everyday use of recovery tools in community mental health, several challenges have arisen. Clients are overwhelmed by the vast number of available digital tools and may not have appropriate computers or smartphones to access them. Clinicians and case managers often lack the technical and practical skills to help their clients use technology to support recovery, and both clients and clinicians need training to use technology collaboratively. Administrators may 
not have the extra funds for a new technology position. While our technology specialists had advanced degrees in mental health, we do not believe that this is necessary. It is likely that case managers or peer specialists may be effective technology specialists after receiving training in the processes of evaluating digital tools and supporting communication regarding technology in routine mental health care. In our opinion, the critical elements of a successful technology specialist are interest in technology, creativity, interpersonal skills, and ability to work collaboratively in a team. The issues of fading interest, clear and measurable outcomes, and emergent ethical concerns have not yet been addressed satisfactorily.

\section{Limitations}

Our preliminary exploration of a new role and intervention has several limitations. The sample was all White/ non-Hispanic, reflecting the demographics in this predominantly White, rural state. We will redress this limitation in future research by partnering with mental health organizations serving a more diverse client population. We did not employ quantitative measures of outcomes; instead, we focused on qualitative interviews in an attempt to understand, at a granular level, client and clinician experiences of the intervention. We saw this as a necessary step not only to obtain information on the feasibility and acceptability of the intervention, but also to begin to understand the ways in which the intervention may be influencing participants. Because of the individualized nature of the intervention and wide range of goals set by participants, selecting appropriate outcomes measures was also a challenge.

\section{Conclusion}

Despite these limitations, our feasibility study suggests that introducing individualized technology tools in community mental health settings has great potential. Future work in this area must address standardization of the intervention, such as creating a manual and training materials; development of guidelines for improving client-clinician collaboration on using technology tools; and identification of appropriate process and outcomes measures. Conducting a larger pilot study is a vital next step. Throughout this process we must address the ways in which the technology specialist role may be adapted into and funded by realworld settings.

Acknowledgements The authors would like to thank the participating clinics and staff who made this project possible. We would especially like to recognize the clients and clinicians who completed the intervention and shared their thoughts, perspectives and expertise with us. We gratefully acknowledge the generous support of this study by the Natalia Mental Health Foundation and the West Family Foundation. Funders had no role in the research activities or the reporting of results.

Funding This study was supported by generous gifts from the Natalia Mental Health Foundation and the West Family Foundation.

\section{Compliance with Ethical Standards}

Conflict of interest The authors report no financial relationships with commercial interests.

\section{References}

Bartels, S. J., Baggett, T. P., Freudenreich, O., \& Bird, B. L. (2020). COVID-19 emergency reforms in massachusetts to support behavioral health care and reduce mortality of people with serious mental illness. Psychiatric Services, 71(10), 1078-1081. https://doi.org/10.1176/appi.ps.202000244.

Ben-Zeev, D. (2020). The digital mental health genie is out of the bottle. Psychiatric Services. https://doi.org/10.1176/appi. ps.202000306.

Ben-Zeev, D., et al. (2014). Feasibility, acceptability, and preliminary efficacy of a smartphone intervention for schizophrenia. Schizophrenia Bulletin, 40(6), 1244-1253. https://doi. org/10.1093/schbul/sbu033.

Ben-Zeev, D., et al. (2017). CrossCheck: Integrating self-report, behavioral sensing, and smartphone use to identify digital indicators of psychotic relapse. Psychiatric Rehabilitation Journal, 40(3), 266-275. https://doi.org/10.1037/prj0000243.

Ben-Zeev, D., et al. (2018). Mobile health (mHealth) versus clinicbased group intervention for people with serious mental illness: A randomized controlled trial. Psychiatric Services, 69(9), 978-985. https://doi.org/10.1176/appi.ps.201800063.

Ben-Zeev, D., Buck, B., Meller, S., Hudenko, W. J., \& Hallgren, K. A. (2020). Augmenting evidence-based care with a texting mobile interventionist: A pilot randomized controlled trial. Psychiatric Services. https://doi.org/10.1176/appi.ps.202000239.

Ben-Zeev, D., Drake, R., \& Marsch, L. (2015). Clinical technology specialists. British Medical Journal, 350, h945. https://doi. org/10.1136/bmj.h945.

Ben-Zeev, D., et al. (2016). mHealth for schizophrenia: Patient engagement with a mobile phone intervention following hospital discharge. JMIR Mental Health, 3(3), e34. https://doi. org/10.2196/mental.6348.

Biagianti, B., Hidalgo-Mazzei, D., \& Meyer, N. (2017). Developing digital interventions for people living with serious mental illness: Perspectives from three mHealth studies. Evidence Based Mental Health, 20(4), 98. https://doi.org/10.1136/eb-2017102765.

Brunette, M. F., et al. (2011). An electronic decision support system to motivate people with severe mental illnesses to quit smoking. Psychiatric Services, 62(4), 360-366. https://doi.org/10.1176/ ps.62.4.pss6204_0360.

Buck, B., Chander, A., \& Ben-Zeev, D. (2020). Clinical and demographic predictors of engagement in mobile health vs. clinicbased interventions for serious mental illness. Journal of Behavioral and Cognitive Therapy, 30(1), 3-11. https://doi. org/10.1016/j.jbct.2020.03.004. 
Carpenter-Song, E. (2020). Promoting meaningful recovery with digital mental health care. Epidemiology and Psychiatric Sciences, 29, e105. https://doi.org/10.1017/S2045796020000165.

Carpenter-Song, E., Noel, V. A., Acquilano, S. C., \& Drake, R. E. (2018). Real-world technology use among people with mental illnesses: Qualitative study. JMIR Mental Health, 5(4), e10652. https://doi.org/10.2196/10652.

Charmaz, K. (2014). Constructing grounded theory. Thousand Oaks, CA: Sage.

Chiauzzi, E., \& Newell, A. (2019). Mental health apps in psychiatric treatment: A patient perspective on real world technology usage. JMIR Mental Health, 6(4), e12292. https://doi.org/10.2196/12292.

Cosgrove, L., Karter, J. M., Morrill, Z., \& McGinley, M. (2020). Psychology and surveillance capitalism: The risk of pushing mental health apps during the COVID-19 pandemic. Journal of Humanistic Psychology, 60(5), 611-625.

Crookston, T. B., et al. (2017). Mental and emotional self-help technology apps: Cross-Sectional study of theory, technology, and mental health behaviors. JMIR Mental Health, 4(4), e45. https ://doi.org/10.2196/mental.7262.

Deegan, P. E. (2010). A web application to support recovery and shared decision making in psychiatric medication clinics. Psychiatric Rehabilitation Journal, 34(1), 23-28. https://doi. org/10.2975/34.1.2010.23.28.

Dugdale, S., Elison-Davies, S., Semper, H., Ward, J., \& Davies, G. (2019). Are computer-based treatment programs effective at reducing symptoms of substance misuse and mental health difficulties within adults? A systematic review. Journal of Dual Diagnosis. https://doi.org/10.1080/15504263.2019.1652381.

Firth, J., et al. (2016). Mobile phone ownership and endorsement of "mHealth" among people with psychosis: A meta-analysis of cross-sectional Studies. Schizophrenia Bulletin, 42(2), 448-455. https://doi.org/10.1093/schbul/sbv132.

Fortuna, K., et al. (2019). Application of community-engaged research to inform the development and implementation of a peer-delivered mobile health intervention for adults with serious mental illness. Journal of Participatory Medicine, 11(1), e12380. https://doi.org/10.2196/12380.

Gagnon, M.-P., Ngangue, P., Payne-Gagnon, J., \& Desmartis, M. (2016). m-Health adoption by healthcare professionals: A systematic review. Journal of the American Medical Informatics Association, 23(1), 212-220. https://doi.org/10.1093/jamia/ ocv052.

Gammon, D., et al. (2017). Shifting practices toward recovery-oriented care through an e-recovery portal in community mental health care: A mixed-methods exploratory study. Journal of Medical Internet Research, 19(5), 14. https://doi.org/10.2196/ jmir.7524.

Garety, P. A., et al. (2017). SlowMo, a digital therapy targeting reasoning in paranoia, versus treatment as usual in the treatment of people who fear harm from others: Study protocol for a randomised controlled trial. Trials, 18(1), 510. https://doi.org/10.1186/s1306 3-017-2242-7.

Gire, N., et al. (2017). mHealth based interventions for the assessment and treatment of psychotic disorders: A systematic review. mHealth, 3, 33-33. https://doi.org/10.21037/mhealth.2017.07.03.

Goldman, M. L., et al. (2020). Mental health policy in the era of COVID-19. Psychiatric Services, 71(11), 1158-1162. https://doi. org/10.1176/appi.ps.202000219.

Hoffman, L., et al. (2020). Digital opportunities for outcomes in recovery services (DOORS): A pragmatic hands-on group approach toward increasing digital health and smartphone competencies, autonomy, relatedness, and alliance for those with serious mental illness. Jounal of Psychiatric Practice, 26(2), 80-88. https://doi. org/10.1097/pra.0000000000000450.
Hubley, S., Lynch, S. B., Schneck, C., Thomas, M., \& Shore, J. (2016). Review of key telepsychiatry outcomes. World Journal of Psychiatry, 6(2), 269-282. https://doi.org/10.5498/wjp.v6.i2.269.

Inkster, B., et al. (2020). Digital health management during and beyond the COVID-19 pandemic: Opportunities, barriers, and recommendations. JMIR Mental Health, 7(7), e19246. https:// doi.org/10.2196/19246.

IQVIA. (2017). The growing value of digital health: Evidence and impact on human health and the healthcare system. Parsippany, NJ: IQVIA Institute for Human Data Science.

Jonathan, G., Carpenter-Song, E. A., Brian, R. M., \& Ben-Zeev, D. (2019). Life with FOCUS: A qualitative evaluation of the impact of a smartphone intervention on people with serious mental illness. Psychiatric Rehabilitation Journal, 42(2), 182-189. https:// doi.org/10.1037/prj0000337.

Jonathan, G., Pivaral, L., \& Ben-Zeev, D. (2017). Augmenting mHealth with human support: Notes from community care of people with serious mental illnesses. Psychiatric Rehabilitation Journal, 40(3), 336-338. https://doi.org/10.1037/prj0000275.

Larsen, M. E., Nicholas, J., \& Christensen, H. (2016). A systematic assessment of smartphone tools for suicide prevention. PLoS ONE, 11(4), e0152285.

Lecomte, T., et al. (2020). Mobile apps for mental health issues: Meta-review of meta-analyses. JMIR mHealth and uHealth, 8(5), e17458. https://doi.org/10.2196/17458.

Mahmoud, H., Vogt, E. L., Dahdouh, R., \& Raymond, M. L. (2020). Using continuous quality improvement to design and implement a telepsychiatry program in rural illinois. Psychiatric Services, 71(8), 860-863. https://doi.org/10.1176/appi.ps.201900231.

Mohr, D. C., Cuijpers, P., \& Lehman, K. (2011). Supportive accountability: A model for providing human support to enhance adherence to ehealth interventions. Journal of Medical Internet Research, 13(1), e30. https://doi.org/10.2196/jmir.1602.

Montori, V. M., Gafni, A., \& Charles, C. (2006). A shared treatment decision-making approach between patients with chronic conditions and their clinicians: The case of diabetes. Health Expectations, 9(1), 25-36. https://doi.org/10.111 1/j.1369-7625.2006.00359.x.

Mueser, K. T., \& Drake, R. E. (2005). How does an intervention become an evidence-based practice? In R. E. Drake, M. R. Merrens, \& D. W. Lynde (Eds.), Evidence-based mental health practice: A textbook (pp. 217-241). New York: WW Norton \& Co.

Naslund, J. A., et al. (2017). Health behavior models for informing digital technology interventions for individuals with mental illness. Psychiatric Rehabilitation Journal, 40(3), 325-335. https:// doi.org/10.1037/prj0000246.

Nicholas, J., Larsen, M. E., Proudfoot, J., \& Christensen, H. (2015). Mobile apps for bipolar disorder: A systematic review of features and content quality. Journal of Medical Internet Research, 17(8), e198.

Noel, V. A., Acquilano, S. C., Carpenter-Song, E., \& Drake, R. E. (2019a). Use of mobile and computer devices to support recovery in people with serious mental illness: Survey study. JMIR Mental Health, 6(2), e12255. https://doi.org/10.2196/12255.

Noel, V. A., Carpenter-Song, E., Acquilano, S. C., Torous, J., \& Drake, R. E. (2019b). The technology specialist: A 21st century support role in clinical care. npj Digital Medicine, 2(1), 61. https://doi. org/10.1038/s41746-019-0137-6.

Pew Research Center. (2018). Mobile fact sheet. Retrieved August 25 , 2020, from http://www.pewinternet.org/fact-sheet/mobile/.

Roberts, L. W., Chan, S., \& Torous, J. (2018). New tests, new tools: Mobile and connected technologies in advancing psychiatric diagnosis. npj Digital Medicine, 1(1), 6. https://doi.org/10.1038/s4174 6-017-0006-0.

Santarossa, S., Kane, D., Senn, Y. C., \& Woodruff, J. S. (2018). Exploring the role of in-person components for online health behavior 
change interventions: Can a digital person-to-person component suffice? Journal of Medical Internet Research, 20(4), e144. https ://doi.org/10.2196/jmir.8480.

Schlosser, D., et al. (2016). Feasibility of PRIME: A cognitive neuroscience-informed mobile app intervention to enhance motivated behavior and improve quality of life in recent onset schizophrenia. JMIR Research Protocols, 5(2), e77. https://doi.org/10.2196/respr ot.5450.

Torous, J., et al. (2018). A hierarchical framework for evaluation and informed decision making regarding smartphone apps for clinical care. Psychiatric Services, 69(5), 498-500. https://doi. org/10.1176/appi.ps.201700423.

Torous, J., Firth, J., Mueller, N., Onnela, J. P., \& Baker, J. T. (2017a). Methodology and reporting of mobile heath and smartphone application studies for schizophrenia. Harvard Review of Psychiatry, 25(3), 146-154. https://doi.org/10.1097/hrp.0000000000 000133.

Torous, J., Jän Myrick, K., Rauseo-Ricupero, N., \& Firth, J. (2020). Digital mental health and COVID-19: Using technology today to accelerate the curve on access and quality tomorrow. JMIR Ment Health, 7(3), e18848. https://doi.org/10.2196/18848.

Torous, J., et al. (2015). Utilizing a personal smartphone custom app to assess the patient health questionnaire-9 (phq-9) depressive symptoms in patients with major depressive disorder. JMIR Mental Health, 2(1), e8. https://doi.org/10.2196/mental.3889.

Torous, J., et al. (2017b). Characterizing smartphone engagement for schizophrenia: Results of a naturalist mobile health study. Clinical Schizophrenia \& Related Psychoses. https://doi.org/10.3371/ csrp.Jtps.071317.
Waalen, J., et al. (2019). Real world usage characteristics of a novel mobile health self-monitoring device: Results from the scanadu consumer health outcomes (SCOUT) study. PLoS ONE, 14(4), e0215468. https://doi.org/10.1371/journal.pone.0215468.

Wisniewski, H., \& Torous, J. (2020). Digital navigators to implement smartphone and digital tools in care. Acta Psychiatrica Scandinavica, 141(4), 350-355. https://doi.org/10.1111/acps.13149.

Wright, J. H., Mishkind, M., Eells, T. D., \& Chan, S. R. (2019). Computer-assisted cognitive-behavior therapy and mobile apps for depression and anxiety. Current Psychiatry Reports, 21(7), 62. https://doi.org/10.1007/s11920-019-1031-2.

Young, A. S., et al. (2020). Mobile phone and smartphone use by people with serious mental illness. Psychiatric Services, 71(3), 280-283. https://doi.org/10.1176/appi.ps.201900203.

Zeuner, R., Frosch, D. L., Kuzemchak, M. D., \& Politi, M. C. (2015). Physicians' perceptions of shared decision-making behaviours: A qualitative study demonstrating the continued chasm between aspirations and clinical practice. Health Expectations, 18(6), 2465-2476. https://doi.org/10.1111/hex.12216.

Zuboff, S. (2019). The age of surveillance capitalism: The fight for a human future at the new frontier of power. New York: PublicAffairs Hachette Book Group.

Publisher's Note Springer Nature remains neutral with regard to jurisdictional claims in published maps and institutional affiliations. 